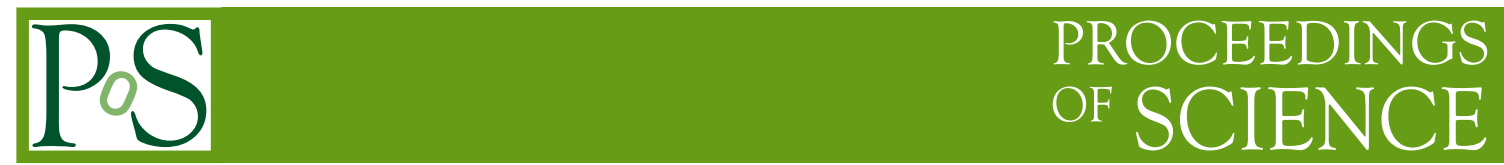

\title{
LHCb status and highlights
}

\author{
Andrey Golutvin*† \\ Imperial College London, ITEP Moscow, CERN \\ E-mail: andrey.goloutvin@cern.ch
}

The status of the LHCb experiment is presented with a particular emphasis on the detector performance validated with collision data.

35th International Conference of High Energy Physics - ICHEP2010,

July 22-28, 2010

Paris France

* Speaker.

†n behalf of the LHCb collaboration 
The $\mathrm{LHCb}$ detector [1] is a forward spectrometer designed for precision studies of CP violation and rare decays of heavy flavours at the Large Hadron Collider at CERN. LHCb successfully started to take data in proton-proton collisions at $\sqrt{s}=7 \mathrm{TeV}$ in March 2010. By the time of the ICHEP2010 conference about $300 \mathrm{nb}^{-1}$ of integrated luminosity has been delivered to experiments. By the time of the submission of this article LHCb has recorded about $37 \mathrm{pb}^{-1}$ of integrated luminosity, which are currently being analysed.

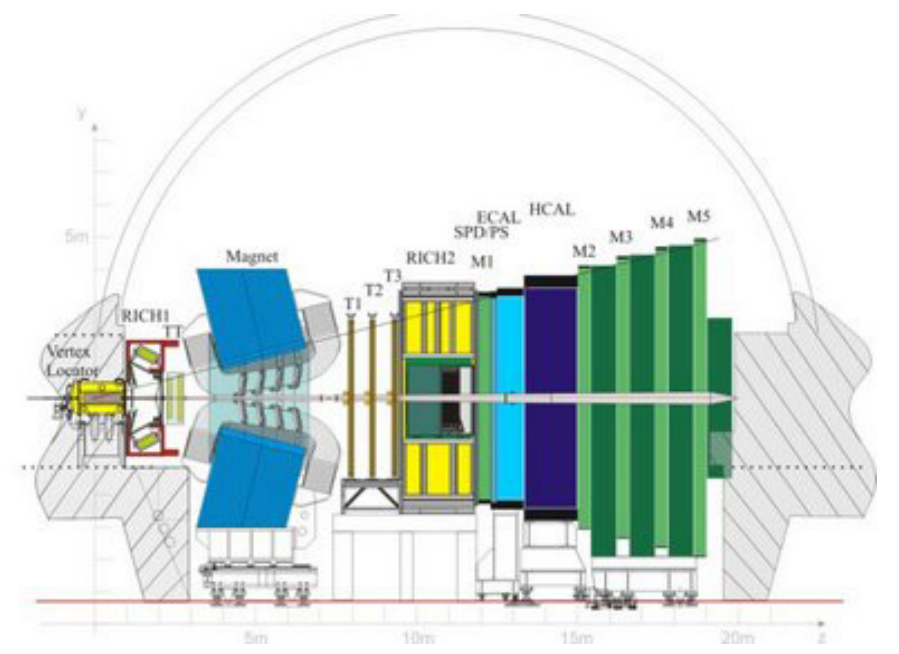

Figure 1: Schematic side view of the $\mathrm{LHCb}$ detector showing the following components: Vertex Locator (VELO), RICH1, Trigger Turicencis (TT), warm dipole magnet, three stations of the main tracker (T1,T2,T3), RICH2, Scintillating Pad Detector (SPD), PreShower (PS), Electromagnetic (ECAL) and Hadronic (HCAL) calorimeters and Muon detector stations (M1 - M5).

\section{Physics objectives for the 2010/2011 Physics run}

The main goal of LHCb physics programme is to search for virtual contribution of New Particles $(N P)$ to Flavour Changing Neutral Currents $(F C N C)$ in reactions mediated by the loop diagrams involving beauty and charm quarks. A search strategy has been developed on an illustrative set of "key measurements" [2] sensitive to the phases and couplings of $N P$ and even to their helicity structure. The LHCb main objectives for 2010/2011 physics run include:

- A measurement of the $B_{s}$ mixing phase, $\phi_{s}$, in $B_{s}^{0} \rightarrow J / \psi(\mu \mu) \phi(K K)$ decays;

- A measurement of the unitarity triangle (UT) angle $\gamma$ in the processes mediated by tree diagrams using both CP-violation time-integrated and time-dependent measurements. These include studies of decays of the type $B^{+} \rightarrow D^{0} K^{+}, B_{d}^{0} \rightarrow D^{0} K^{* 0}, B_{d}^{0} \rightarrow D \pi$ and $B_{s}^{0} \rightarrow D_{s} K$;

- A measurement of the UT angle $\gamma$ in loop mediated processes. The $B_{d, s}^{0} \rightarrow h^{+} h^{-}$family of decays, where $h$ stands for a pion or kaon, has decay rates with sizable contribution from penguin diagrams, making them sensitive to NP effects in penguin loops;

- A search for the super-rare $B_{s}^{0} \rightarrow \mu^{+} \mu^{-}$decay; 
- A test of the $V-A$ helicity structure of the weak interaction in loop mediated $B_{d}^{0} \rightarrow K^{* 0} \mu^{+} \mu^{-}$ decays;

- A search for CP-violation asymmetries in charm decays.

\section{Validation of the detector performance using data}

The key features of LHCb performance are excellent vertex and impact parameter resolution, high track reconstruction and particle identification [3, 4] efficiency and a flexible trigger [5] which effectively selects particles produced in the decays of heavy flavours.

\subsection{Reconstruction and Particle Identification performance}

Compared to the other LHC experiments, in the months prior to first collisions LHCb could only benefit to a very limited extent from using cosmic rays due to the forward geometry of the spectrometer. Therefore much attention has been paid during the 2010 run to validate the detector performance with collision data. Modest discrepancies compared with Monte Carlo expectations have been observed in a few places. These imperfections are being addressed with the highest priority and a continuous improvement in the understanding of detector performance is ongoing. In order to demonstrate the impressive progress achieved since summer 2010 some of the key performance figures shown at the conference are here replaced with the most recent updates.

Excellent vertexing capabilities and proper time resolution are provided by the Vertex Locator (VELO), the detectors of which approach to within $8 \mathrm{~mm}$ of the beam line during collisions. The sensors of the VELO have been aligned to an accuracy of a few $\mu \mathrm{m}$ using tracks. The individual hit resolution of the sensors is a strong function of the sensor pitch and projected angle (the angle perpendicular to the strip direction), as shown in Figure 2(left). A single hit resolution of $4 \mu \mathrm{m}$ has been achieved at optimal projected track angle for the smallest pitch size. The large impact
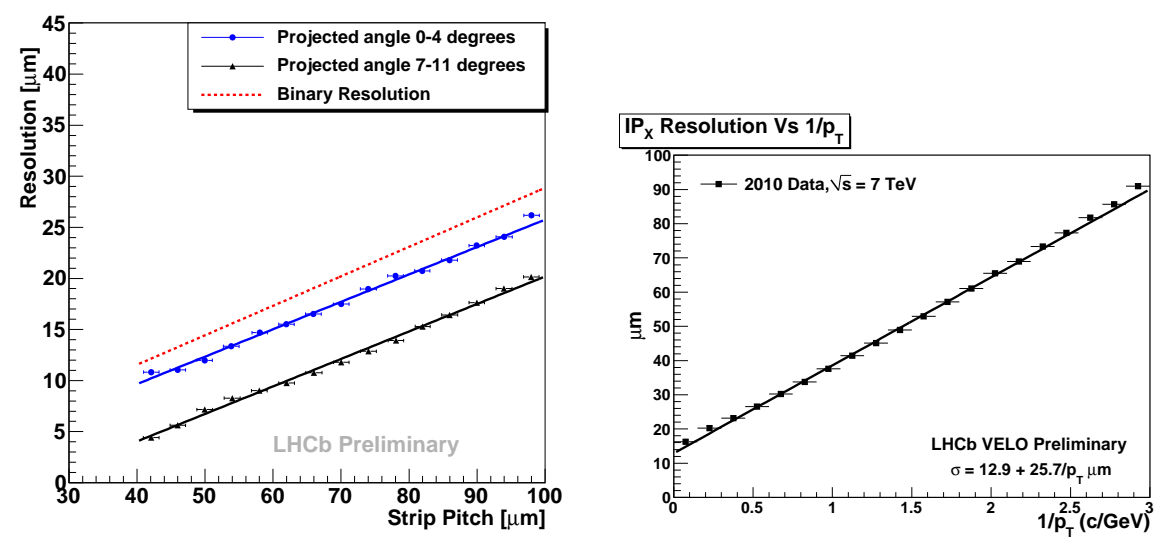

Figure 2: The left hand histogram shows the VELO hit resolution versus the strip pitch in two projected angle regions. The right hand histogram shows the $I P_{X}$ resolution as a function of $1 / P_{T}$.

parameter $(I P)$ of the track with respect to the interaction vertex is a distinctive feature to identify heavy flavour particle decays. The impact parameter resolution currently achieved is shown in 
Figure 2(right) in bins of $1 / P_{t}$ of the track. The resolution of $14 \mu \mathrm{m}$ in the highest $P_{t}$ bin agrees very well with the Monte Carlo expectation. Slightly different slopes observed in data and Monte Carlo resolution, not shown in Figure 2 (right), as a function of $1 / P_{t}$ probably indicate at yet not perfect description of material distribution in the Monte Carlo. Studies are underway with data to map out the amount and distribution of material by reconstructing hadronic secondary interactions in the VELO. Closely related to the $I P$ resolution is the vertex resolution which is a quantity crucial for the proper time reconstruction and for separating vertices from multiple interactions. Vertex resolutions have been measured by randomly splitting all reconstructed tracks into two subsets and by reconstructing vertices from each of the subsets. For a typical vertex producing 25 tracks the resolution has been found to be $15 \mu \mathrm{m}$ in $X$ and $Y$, and $75 \mu \mathrm{m}$ in $Z$. Further improvement is expected with advances on the alignment and track reconstruction.

Good progress has been achieved on the alignment of the tracking detectors, the Tracker Turicencis (TT) and three stations of the main tracker (T1, T2, T3) (see Figure 11), using data with reconstructed muons from $J / \psi$ decays. Cluster resolutions of $55 \mu \mathrm{m}$ have been obtained for the silicon microstrip detectors in both the TT and Inner Tracker (IT) which occupies the inner parts of the T1, T2 and T3 stations. The measured values are consistent with the expected binary resolution of the detectors. For the outer part of the T1, T2 and T3 stations, which are made of $5 \mathrm{~mm}$ diameter Kapton/Al straw tubes, a spatial resolution of $250 \mu \mathrm{m}$ has been measured with data. Further improvement is expected after better alignment of the spectrometer.

A continuous progress in the spectrometer alignment and calibration of momentum resolution is reflected in improved agreement between data and Monte Carlo for the invariant mass resolution measured for selected benchmark decays. The most recent alignment has significantly improved the invariant mass resolutions for $D$ and $B$ mesons (Figure 3 ), and for the $J / \psi$ and $\Upsilon$ resonances (Figure 47). The measured resolutions are now twice narrower than was the case in ICHEP 2010 conference and very close to the Monte Carlo expectation.

In the track reconstruction algorithm the hits in the VELO, TT, IT and OT detectors are combined to form particle trajectories from the VELO to the calorimeter system. Several methods have been implemented to assess the tracking efficiency on data. In particular $K_{S}^{0} \rightarrow \pi^{+} \pi^{-}$candidates have been reconstructed from one existing long track, leaving hits in all tracking sub-detectors for one of the daughters, and the VELO track pointing to the calorimeter cluster for the second daughter. The efficiency of long tracks is defined by the integral of the $K_{S}^{0}$ peak for cases where the VELO track is used in a long track which points to the calorimeter cluster normalised by the integral of the peak without the long track association. The efficiency has been found to be $95 \%$ for all long tracks with $P_{T}$ above $100 \mathrm{MeV} / \mathrm{c}$ which is in agreement with Monte Carlo expectation in the full physics range of $\mathrm{LHCb}$ within $\sim 4 \%$, as shown in Figure 5 (left). A similar method has been applied to evaluate the efficiency of the track reconstruction in VELO.

The track reconstruction efficiency can be also evaluated using the ratio of the branching ratios for well measured decay channels, which result in final states with different number of particles, such as $D^{0} \rightarrow K \pi$ and $D^{0} \rightarrow K \pi \pi \pi$. The ratio of the corresponding rates is proportional to the track reconstruction efficiency squared: $\varepsilon($ track $) \propto \sqrt{\frac{N(K \pi \pi \pi)}{N(K \pi)} \times \frac{B R(K \pi)}{B R(K \pi \pi \pi)}}$. Using this method the ratio of the efficiencies obtained with data and Monte Carlo has been found to be $1.03 \pm 0.03$.

Excellent identification of leptons and hadrons is fundamental for the LHCb physics pro- 

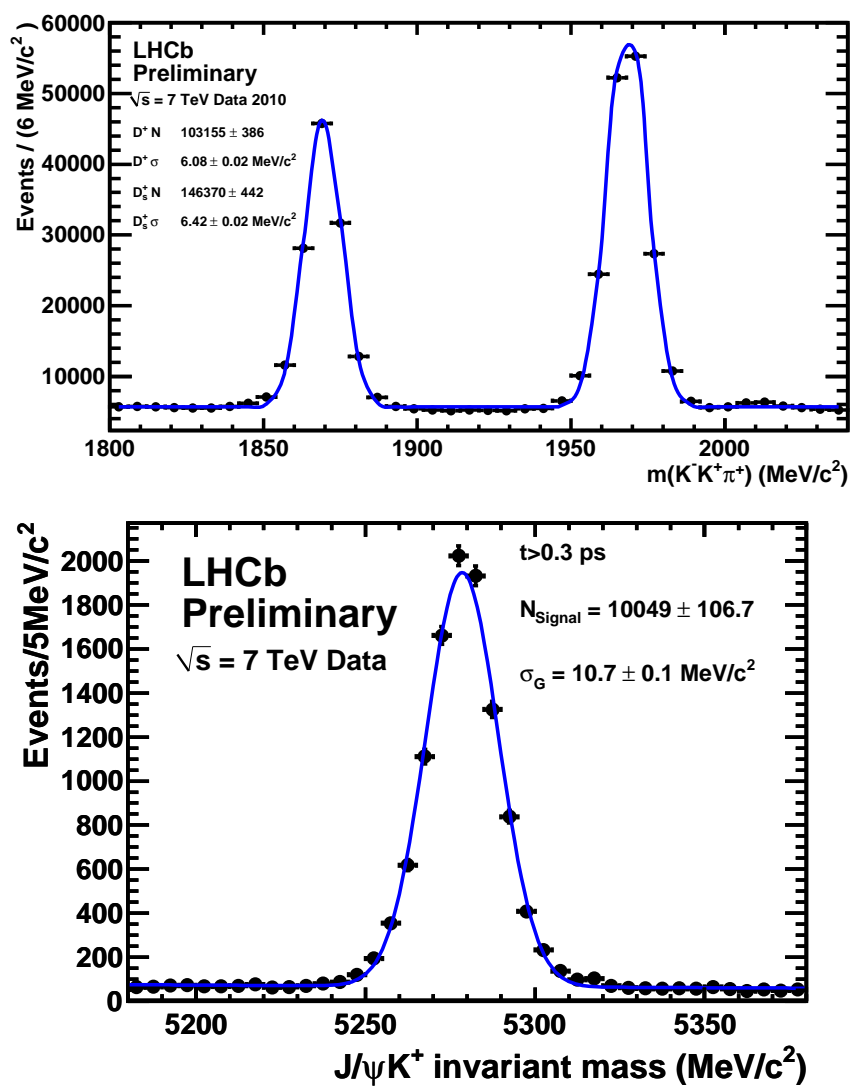

Figure 3: The top histogram plots the $K^{-} K^{+} \pi^{+}$invariant mass showing the signals from $D^{+}$and $D_{s}$ decays. The bottom histogram shows the $B^{+}$signal in $J / \psi K^{+}$invariant mass.

gramme. Pion, kaon and proton identification is based on using the information from two RICH detectors which contain three different radiators, silica aerogel and the $C_{4} F_{10}$ gas in RICH1, and $\mathrm{CF}_{4}$ gas in $\mathrm{RICH} 2$. Combining the two radiators RICH1 provides a separation between kaons and pions for momenta up to $50 \mathrm{GeV} / \mathrm{c}$. Particles with higher momenta up to $100 \mathrm{GeV} / \mathrm{c}$ are identified using RICH2. The performance of pion and proton identification has been studied using the daughter particles of reconstructed $K_{S}$ mesons and $\Lambda$ baryons. For the kaons, the reconstructed $\phi \rightarrow K^{+} K^{-}$decays have been used. Identifying only a single track using RICH information, and leaving the other unbiased, yields a sufficiently pure sample that can be used to measure the kaon identification efficiency. We now also use kaons from $D^{0} \rightarrow K \pi$ decay channel in $D^{*}$ decays.

The $K / \pi$ and $p / \pi$ separation performance achieved currently is shown in Figures 5 (right). The best performance is achieved in the momentum range from 5 to $70 \mathrm{GeV} / \mathrm{c}$ where most hadrons from $b$ decays are produced.

Electron and photon identification is mainly based on the balance of the energy deposited in the calorimeter system and the track momentum, and the matching between the corrected barycenter position of the calorimeter cluster and the extrapolated track impact point. The electromagnetic calorimeter has been calibrated to $\sim 2 \%$ accuracy resulting in a $\pi^{0}$ invariant mass resolution of $\sim 7 \mathrm{MeV} / \mathrm{c}^{2}$ as shown in Figure 6 (left). Using the electrons produced in photon conversions the 

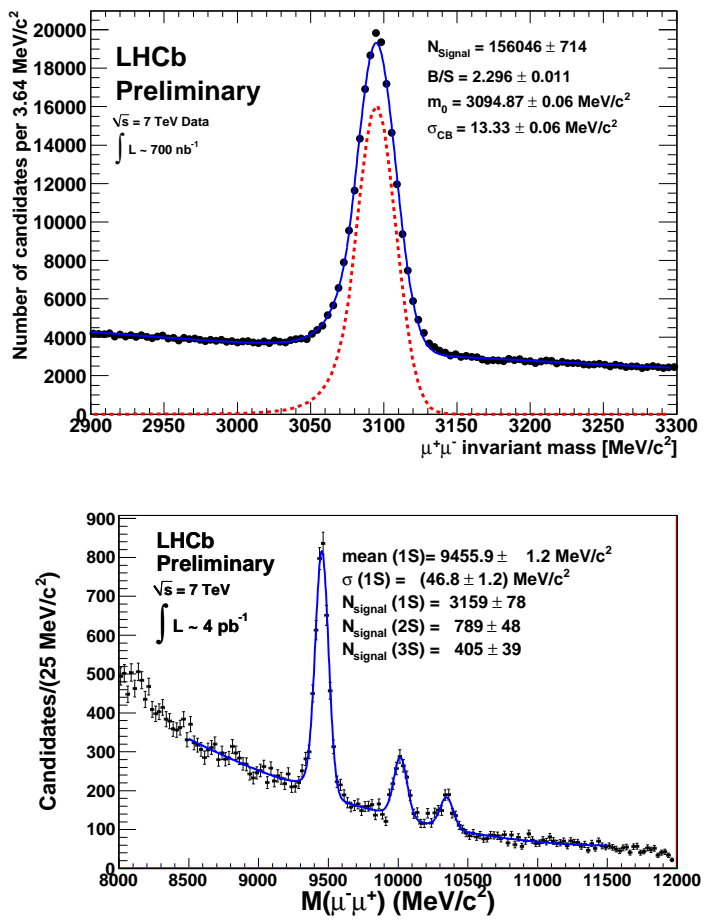

Figure 4: The $\mu^{+} \mu^{-}$invariant mass distribution in the $J / \psi$ (top) and $\Upsilon$ (bottom) regions.
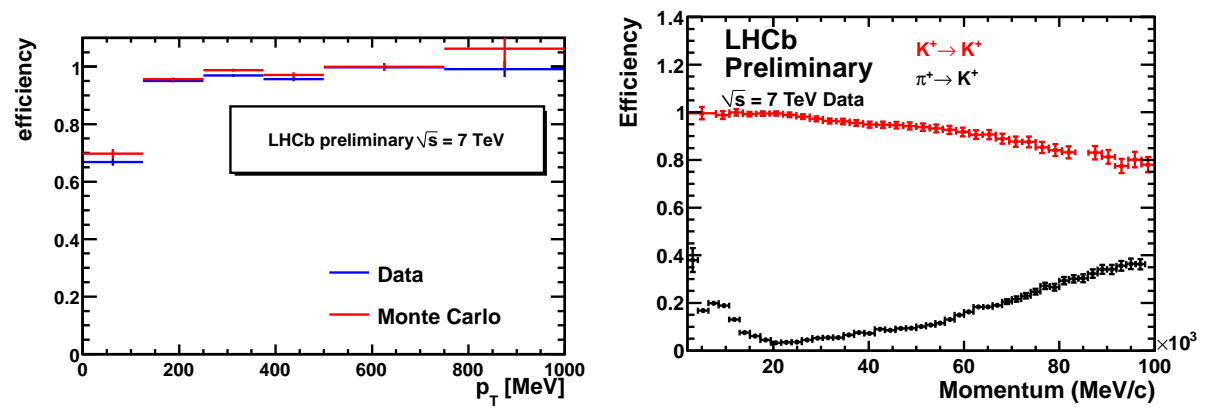

Figure 5: The left hand histogram shows the track reconstruction efficiency as a function $P_{t}$. The right hand histogram shows the RICH $\pi / K$ separation performance as a function of momentum.

electron identification efficiency has been found to be $\geq 90 \%$ with misidentification rate of $\sim 3-5 \%$ for the electrons with momentum above $10 \mathrm{GeV} / \mathrm{c}$.

Muons are identified by extrapolating well reconstructed tracks with $P \geq 3 \mathrm{GeV} / \mathrm{c}$ into the muon stations and matching the tracks with the hits within the corresponding fields of interest. Using a sample of reconstructed $J / \psi \rightarrow \mu^{+} \mu^{-}$decays the muon identification efficiency has been measured to be $(97.3 \pm 1.2) \%$ which is in a good agreement with Monte Carlo expectation. As shown in Figure 6(right), the efficiency is a flat function of momentum above $10 \mathrm{GeV} / \mathrm{c}$. Corresponding $\mu / \pi$ and $\mu / K$ misidentification rates are dominated by the $\pi, K$ decays in flight and found to be below $1 \%$ for $P>20 \mathrm{GeV} / \mathrm{c}$. 

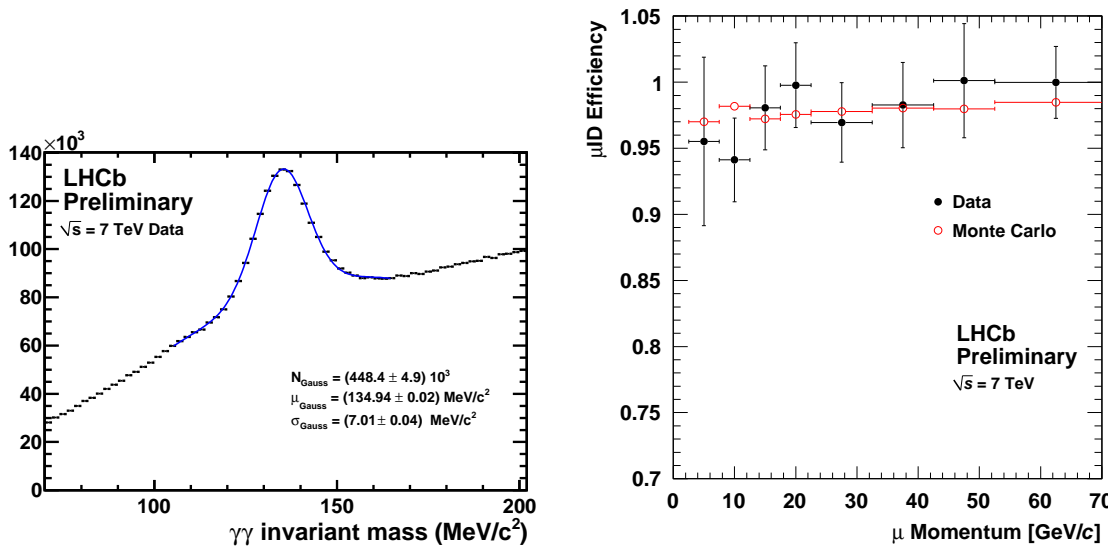

Figure 6: The left hand histogram shows the invariant mass of two photons reconstructed in the calorimeter system. The right hand histogram shows the muon identification efficiency as a function of muon momentum.

\subsection{Trigger performance}

For the best overall performance the LHCb trigger is optimised to achieve the highest efficiency for the events selected in the off-line analysis while reducing the background from the input rate of about $40 \mathrm{MHz}$ down to $2 \mathrm{KHz}$, at which rate the events are written to storage for subsequent off-line analysis. This reduction is achieved in two levels: a hardware implemented Level-0 ( $L 0)$ and a software based High Level Trigger (HLT). The purpose of $L 0$ is to reduce the input rate down to $1 \mathrm{MHz}$, at which value the entire detector can be read out, by selecting events with the highest $P_{T}$ signals in the calorimeter and muon systems. In order to further reduce the event rate from $1 \mathrm{MHz}$ down to $2 \mathrm{kHz}$, the $H L T$ makes use of the full event information. After refining the candidates found by $L 0$, events are divided into a mixture of inclusive and exclusive channels. At the LHCb designed luminosity $\left(2 \times 10^{32} \mathrm{~cm}^{-2} \mathrm{~s}^{-1}\right)$ the trigger thresholds have to be optimised for $B$-physics, and consequently the trigger efficiency for $D$ decays from prompt production is as low as $\sim 10 \%$, which is a factor of $\sim 4$ lower than the corresponding efficiency for $B$ decays. For the bulk of running foreseen in 2010, with luminosity up to a few $\times 10^{30} \mathrm{~cm}^{-2} \mathrm{~s}^{-1}$, many of the trigger thresholds could be relaxed in order to increase the efficiency for hadronic $D$ and $B$ decays up to $\sim 40 \%$ and $\sim 70 \%$ respectively. The trigger efficiency for leptonic decay modes has been found to be higher than $90 \%$. The muon trigger performance has been validated on data using lifetime unbiased selection for $J / \psi \rightarrow \mu^{+} \mu^{-}$decays. As shown in Figure 7 data and Monte Carlo expectation agree very well providing an important proof of LHCb trigger concept.

\section{Measurements of production cross-sections}

The measurement of beauty and charm production cross-setions at $\sqrt{s}=7 \mathrm{TeV}$ in the detector acceptance is of direct importance in ascertaining the sensitivities for the fundamental parameters of LHCb physics programme. Furthermore the knowledge of double-differential cross-sections as a function of $P_{T}$ and rapidity $y$ has its own interest in order to test theoretical models. An estimate of integrated luminosity, needed for the cross section determination, has been obtained 

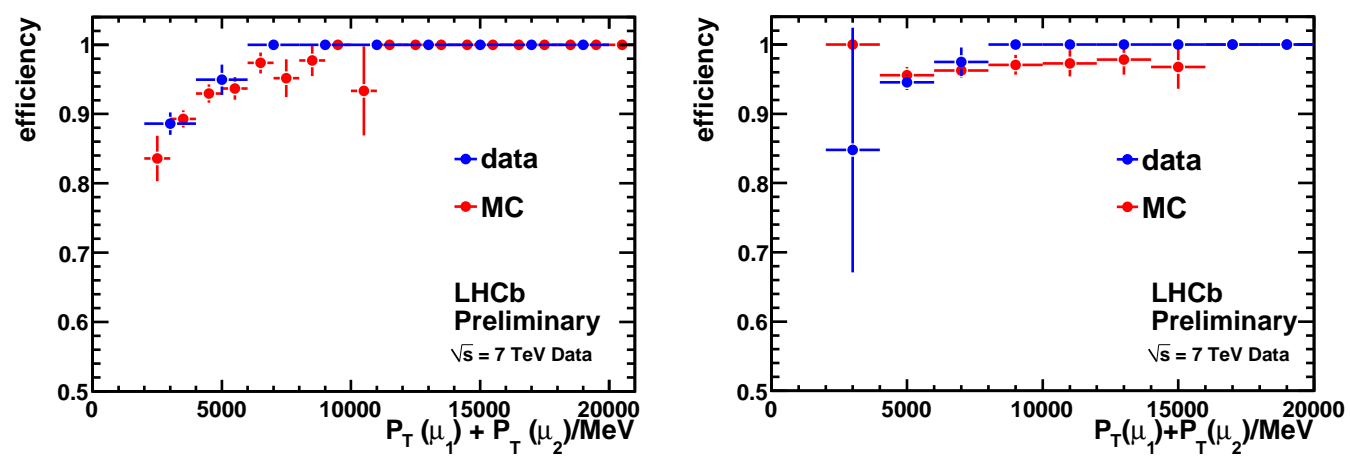

Figure 7: $L 0 \times H L T$ efficiency for $J / \psi$ selection (left) and $B_{s} \rightarrow \mu^{+} \mu^{-}$selection (right).

using Van der Meer scan and a "beam imaging" technique developed specifically at LHCb [6]. Since the total accuracy is dominated by the systematic error on the luminosity measurement and track reconstruction efficiency, only small sub-sample of collected data, including those taken with unbiased trigger, has been used in these analyses.

\subsection{Beauty production cross-section}

The $b$ production cross section has been measured by two methods $[7,8 \rrbracket$ using samples of inclusive $B \rightarrow J / \psi X$ and semileptonic $B \rightarrow \bar{D} X \ell v$ decays. The impact parameter (IP) for $D$ mesons and pseudo proper time $t_{z}{ }^{1}$ for $J / \psi$ mesons have been used to separate mesons produced directly in $p p$ collisions and in $B$ decays.

The fraction of $J / \psi$ produced in $B$ decays, $f_{b}=11.1 \pm 0.8 \%$, has been determined from a simultaneous fit to $\mu^{+} \mu^{-}$invariant mass and $t_{z}$ distributions shown in Figure 8 for selected events. Taking into account systematic uncertainties this value of $f_{b}$ corresponds to the following total
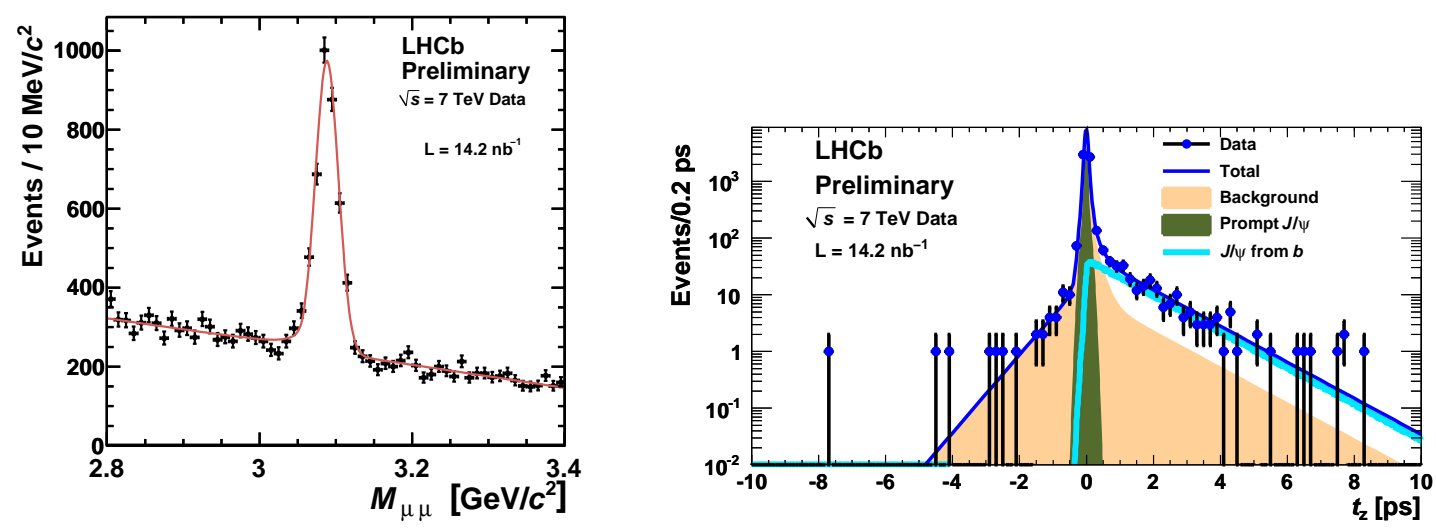

Figure 8: The left hand distribution shows dimuon invariant mass for the $J / \psi \rightarrow \mu^{+} \mu^{-}$signal used for the cross-section measurement. The right hand distribution shows the pseudo-proper time $t_{z}$ for the $J / \psi$ candidates.

\footnotetext{
${ }^{1} t_{z}=d_{z} \times M / P_{z}$, where $d_{z}$ and $P_{z}$ are the $z$-axis components of the distance between the $J / \psi$ decay vertex and primary vertex
} 
cross-section for inclusive $J / \psi$ production in $B$ decays

$$
\sigma\left(J / \psi \text { from } b, P_{t}<10 \mathrm{GeV} / c, 2.5<y<4\right)=0.81 \pm 0.06 \pm 0.13 \mu b
$$

in the detector acceptance. Using the LHCb Monte Carlo based on PYTHIA 6.4 and EvtGen this measurement can be extrapolated to the average cross-section of $b$ or $\bar{b}$ hadron production, $H_{b}$, in the pseudorapidity region covered by $\mathrm{LHCb} 2<\eta<6$ and to the full solid angle acceptance

$$
\sigma\left(p p \rightarrow H_{b} X, 2<\eta\left(H_{b}\right)<6\right)=84.5 \pm 6.3 \pm 15.6 \mu b ; \sigma(p p \rightarrow b \bar{b} X)=319 \pm 24 \pm 59 \mu b
$$

Inclusive semileptonic decays $B \rightarrow \bar{D}^{0} X \ell v$ followed by $\bar{D}^{0} \rightarrow K^{-} \pi^{+}$are cleanly reconstructed at $\mathrm{LHCb}$ using the correlation between the signs of the kaon and lepton charges as shown in Figure 9. For the right sign combination expected in semileptonic $B$ decays a pronounced $D$ signal is seen in Figure 9 (left) whilst the $K^{-} \pi^{+}$invariant mass distribution shows no evidence for a $D^{0}$ signal in the wrong sign combinations (Figure 9 (right)). The yield of $D^{0}$ mesons produced in $B$ decays has been determined from the fit of the two-dimensional distributions in $K^{-} \pi^{+}$invariant mass and $D^{0} \ln (I P) / \mathrm{mm}$. The corresponding $\sigma\left(p p \rightarrow H_{b} X\right)$ cross-section in the detector acceptance $2<\eta<6$ has been found to be $74.9 \pm 5.3 \pm 12.8 \mu \mathrm{b}$. Using the same extrapolation procedure
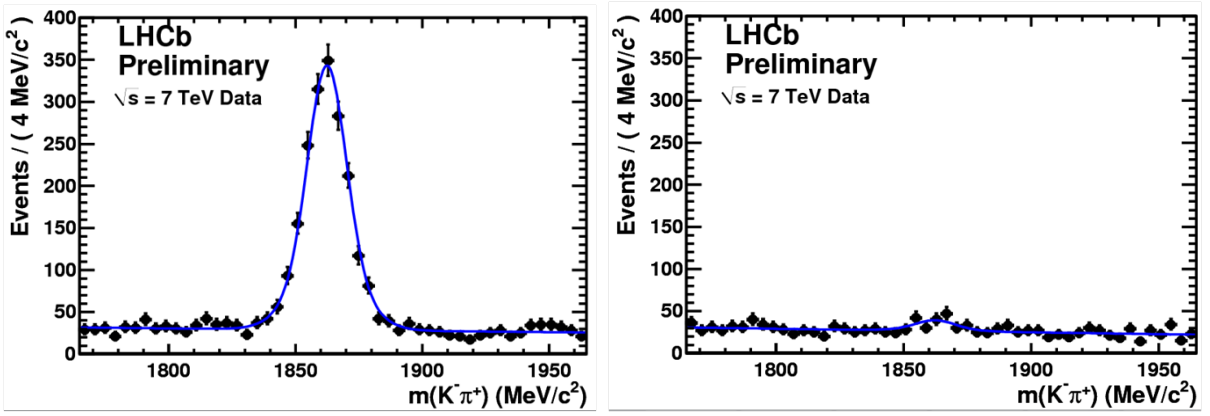

Figure 9: $D^{0} \rightarrow K^{-} \pi^{+}$invariant mass for $D^{0} \mu$ candidates in the right-sign (left) and wrong-sign (right) combinations.

as for $b \rightarrow J / \psi X$ production, a total $b \bar{b}$ cross section in the full solid angle acceptance equals to $282 \pm 20 \pm 48 \mu b$. Both methods of the $b \bar{b}$ production cross-section measurement are in excellent agreement. Averaging between them using the LEP $b$ hadronization fractions gives

$$
\sigma\left(p p \rightarrow H_{b} X, 2<\eta\left(H_{b}\right)<6\right)=79.1 \pm 4.0 \pm 11.4 \mu b ; \sigma(p p \rightarrow b \bar{b} X)=298 \pm 15 \pm 43 \mu b .
$$

The $b \bar{b}$ cross-section was also measured using $B \rightarrow D^{*} \mu v X$ decays $[9$, in good agreement with the other two approaches.

\subsection{Charm production cross-section}

The mechanism of the direct $J / \psi$ production in hadron collisions, including feed-down from the decay of heavier prompt $c \bar{c}$ states, is still not well understood. The prompt $J / \psi$ production is seen as a delta-function at zero value of $t_{z}$ distribution shown in Figure 8 (right). The corresponding differential cross-sections has been determined in 10 bins of $P_{t}$ in the interval $0<P_{t}<10 \mathrm{GeV} / \mathrm{c}$, integrating over the rapidity range $2.5<y<4$ [7]. Results are presented in Figure 10 (top) in 
comparison with Monte Carlo expectations based on colour singlet and colour octet models as implemented in the LHCb simulation. The total prompt $J / \psi$ cross-section in the detector acceptance has been found to be

$$
\sigma\left(p p \rightarrow J / \psi X, P_{t}<10 \mathrm{GeV} / c, 2.5<y<4\right)=7.65 \pm 0.19 \pm 1.10_{-1.27}^{+0.87} \mu b,
$$

where the third error is due to efficiency dependence on $J / \psi$ polarization.
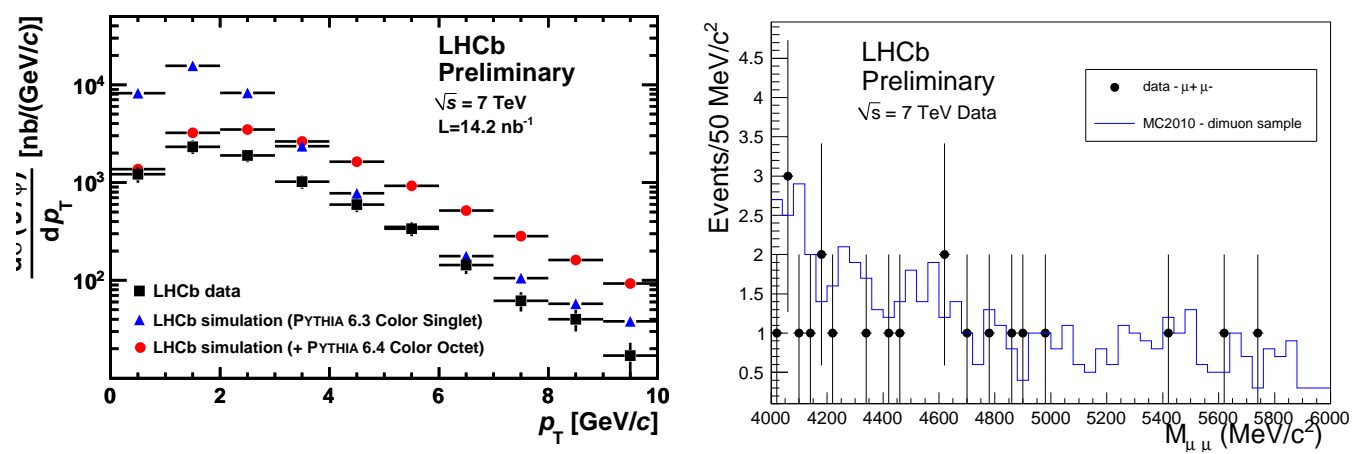

Figure 10: The top distribution shows measured cross-section of prompt $J / \psi$ production compared to Monte Carlo simulations based on colour singlet and colour octet models. The bottom distribution shows $\mu^{+} \mu^{-}$invariant mass for the events survived the selection to search for $B_{s} \rightarrow \mu^{+} \mu^{-}$decay. A data sample corresponds to $215 \mathrm{nb}^{-1}$ of integrated luminosity.

The open charm production cross-sections have been measured for the following decay channels $D^{0} \rightarrow K^{-} \pi^{+}, D^{*+} \rightarrow D^{0}\left(K^{-} \pi^{+}\right) \pi^{+}, D^{+} \rightarrow K^{-} \pi^{+} \pi^{+}$and $D_{s}^{+} \rightarrow \phi\left(K^{+} K^{-}\right) \pi^{+}$[10] in the accessible phase space $0<P_{t}<8 \mathrm{GeV} / \mathrm{c}$ and $2<y<4.5$. All measurements are in good agreement with theory predictions and with the PYTHIA based LHCb Monte Carlo. Using $c$ hadronization fractions the individual cross-section can be translated into the total $c \bar{c}$ production cross-section. Applying the same procedure for extrapolation to the full solid angle acceptance as for the $b \bar{b}$ cross-section, one gets $\sigma(p p \rightarrow c \bar{c})=6100 \pm 934 \mu b$, which is a factor $>20$ higher than the $b \bar{b}$ production cross-section.

\section{Prospects for the key measurements}

The data sample of $\sim 180 \mathrm{nb}^{-1}$ accumulated by the time of ICHEP 2010 was sufficient to observe significant signal peaks in a large fraction of the key decay modes of the LHCb physics programme [10, 11, 12, 13, 14]. Hadronic decay modes of $B$ mesons with branching ratios of $10^{-5}$ have been observed. The event yields are similar to those expected from simulation, showing the trigger and tracking are performing as expected. The background level is satisfactory in all cases. For example, Figure 10 (bottom) shows the distribution on $\mu^{+} \mu^{-}$invariant mass for the events that passed the selection developed to search for $B_{s} \rightarrow \mu^{+} \mu^{-}$decay. Data agree very well with the level of background expected in the Monte Carlo for the collected integrated luminosity of $215 \mathrm{nb}^{-1}$.

The current LHC plan foresees a two years physics run at the centre-of-mass energy of $\sim 7 \mathrm{TeV}$ in order to collect a data sample of $\sim 1 \mathrm{fb}^{-1}$ integrated luminosity. LHCb plans for early physics 
studies will evolve in accordance with the integrated luminosity delivered by LHC. High class measurements for the study of $C P$ violation in the charm sector are feasible with $100 \mathrm{pb}^{-1}$ of integrated luminosity. Based on the currently observed yields we expect several millions of tagged $D^{0} \rightarrow K^{+} K^{-}$decays which can be used to achieve $0.1 \%$ sensitivity in the $A_{\Gamma}$ measurement. A sample of several million $D^{+} \rightarrow K^{+} K^{-} \pi^{+}$decays, which receives significant contribution from gluonic Penguin diagrams, should allow an improvement in the world sensitivity on the search for direct $C P$ violation. The "golden" LHCb measurements of the mixing phase, $\phi_{S}$ [13] and the search for the $B_{s} \rightarrow \mu^{+} \mu^{-}$decay mode [14] will certainly reach world best sensitivity with $100 \mathrm{pb}^{-1}$ of integrated luminosity, opening exciting prospects for discoveries in 2011.

\section{References}

[1] The LHCb Collaboration, A.A. Alves et al., The LHCb Detector at LHC, JINST 3 (2008) S08005.

[2] The LHCb Collaboration, B. Adeva et al., arXiv:0912.4179v2 [hep-ex].

[3] S. Borghi, Performance of the tracking system at the LHCb experiment, these proceedins.

[4] A. Powell, Particle ID in LHCb, these proceedings.

[5] E. Van Herwijen, LHCb trigger system.

[6] M. Ferro-Luzzi, Proposal for an absolute luminosity determination in colliding beam experiments using vertex detection of beam-gas interactions, Nucl. Instr. Meth. A 553 (2005) 388; M. Ferro-Luzzi, Determination of the luminosity by the LHC experiments, these proceedings.

[7] G. Passaleva, Prompt $J / \psi$ and $b \rightarrow J / \psi X$ production in pp collisions at $\sqrt{s}=7 \mathrm{TeV}$, these proceedings; The LHCb Collaboration, Measurement of the $J / \psi$ production cross-section at $\sqrt{s}=7 \mathrm{TeV}$ in LHCb, LHCb-CONF-2010-010 (2010).

[8] S. Stone, First Physics Results from $\mathrm{LHCb}$, these proceedings; The LHCb Collaboration, R. Aaij et al., Measurement of $\sigma(p p \rightarrow b \bar{b} X)$ at $\sqrt{s}=7$ TeV in the forward region, Physics Letters B 694 (2010) 209.

[9] The LHCb Collaboration, Measurements of $B^{0}$ mesons production cross-section in pp collisions at $\sqrt{s}=7 \mathrm{TeV}$ using $B^{0} \rightarrow D^{*} \mu^{+} v_{\mu} X$ decays, LHCb-CONF-2010-012.

[10] I. Belyaev, Results and prospects for Charm Physics at LHCb; The LHCb Collaboration, Prompt charm production in pp collisions at $\sqrt{s}=7 \mathrm{TeV}$, LHCb-CONF-2010-013.

[11] S. Haines, Studies of charmed hadronic B decays with early LHCb data and prospects for $\gamma$ measurement, these proceedings.

[12] I. De Bediaga Hickman, Prospects for CP violation measurements with charmless hadronic B meson decays at $\mathrm{LHCb}$, these proceedings.

[13] G. Raven, Prospects for CP violation in $B_{s}^{0} \rightarrow J / \psi \phi$ from first $L H C b$ data, these proceedings.

[14] G. Mancinelli, Search for New Physics with Rare Heavy Flavour Decays at LHCb, these proceedings. 